\title{
A SUPERVISÃO COMO ESTRATÉGIA DE ACOMPANHAMENTO E AVALIAÇÃO DAS PRÁTICAS DAS EQUIPES DE SAÚDE DA FAMÍLIA
}

\author{
Michela Macedo Lima Costa ${ }^{1}$, Marília Santos Fontoura²
}

\begin{abstract}
RESUMO: Trata-se de um estudo teve como objetivo descrever o processo de supervisão desenvolvido pela Coordenação da Atenção Básica em relação ao processo de trabalho das equipes Saúde da Família do Sudoeste baiano. Caracteriza-se como pesquisa de abordagem qualitativa, com uso da técnica da triangulação das informações decorrentes da pesquisa documental, entrevistas e do grupo focal com os profissionais, e foi realizada em 2008. 008. Pode-se observar que a supervisão favorece a proximidade e o estabelecimento de vínculos como aspectos favoráveis ao desenvolvimento e avaliação do processo de trabalho. Neste estudo, a atividade de supervisão apresentou-se como uma ferramenta de gestão que demanda planejamento, envolvimento, interação, continuidade e corresponsabilidade. Nessa direção, é importante que seja construída num esforço conjunto entre gestores, profissionais de saúde e usuários.
\end{abstract}

PALAVRAS-CHAVE: Saúde da família; Supervisão de enfermagem; Trabalhadores.

\section{SUPERVISION AS A STRATEGY FOR MONITORING AND EVALUATION OF FAMILY HEALTH TEAM PRACTICES}

ABSTRACT: This study aimed to describe the process of supervision developed by the Primary Care Coordination Group in relation to the Family Health teams' work in the Bahian Southwest. This research, carried out in 2008, has a qualitative approach, using the technique of triangulation of the information resulting from a documental survey, interviews, and from a focus group with the professionals. It may be seen that supervision benefits closeness and the establishing of links with aspects favorable to the development and evaluation of the work process. In this study, the activity of supervision was shown as a management tool which requires planning, involvement, interaction, continuity, and co-responsibility. In this sense, it is important that it should be constructed in a joint effort between managers, health professionals and service users. KEYWORDS: Family health; Nursing, supervisory; Workers.

\section{LA SUPERVISIÓN COMO ESTRATEGIA DE ACOMPAÑAMIENTO Y EVALUACIÓN DE LAS PRÁCTICAS DE LOS EQUIPOS DE SALUD DE LA FAMILIA}

RESUMEN: La finalidad de este estudio fue describir el proceso de supervisión desarrollado por la Coordinación de la Atención Básica acerca del proceso de trabajo de los equipos Salud de la Familia del Sudoeste baiano. Se caracteríza como investigación de abordaje cualitativo, cuya técnica utilizada fue de la triangulación de las informaciones originadas de la investigación documental, entrevistas y de grupo focal con los profesionales. Fue realizada en el año de 2008. Lo que se puede observar es que la supervisión favorece la proximidad y el establecimiento de vínculos como aspectos favorables al desarrollo y evaluación del proceso de trabajo. En este estudio, la actividad de supervisión se presentó como una herramienta de administración que demanda planeamiento, participación, interacción, continuidad y corresponsabilidad. Así, es importante que se construya en un esfuerzo conjunto entre gestores, profesionales de salud y usuarios.

PALABRAS CLAVE: Salud de la familia; Supervisión de enfermería; Trabajadores.

\footnotetext{
${ }^{1}$ Enfermeira. Mestre em Saúde Coletiva. Professora da Faculdade Independente do Nordeste.

${ }^{2}$ Enfermeira. Doutora em Saúde Coletiva. Professora do Curso de Graduação da Escola de Enfermagem da Universidade Federal da Bahia.
} 


\section{INTRODUÇÃO}

A Estratégia Saúde da Família (ESF) tem sido apresentada pelo Ministério da Saúde como uma estratégia para consolidação do Sistema Único de Saúde e é reconhecida como alternativa para reversão do modelo assistencial vigente. Nela, a reorganização nas práticas de trabalho em saúde é um desafio para a mudança do atual modelo de assistência em saúde.

Para a construção de um determinado modelo de atenção, a ESF se alicerça na mudança do objeto de atenção, na transformação das práticas assistenciais desenvolvidas pelos profissionais e da organização dos serviços $^{(1)}$. Nessa perspectiva, cabe aos profissionais da atenção básica desenvolver ações voltadas para a promoção da saúde, prevenção de agravos, tratamento das enfermidades, e também o reconhecimento e o diagnóstico do território da área de abrangência, articulação das ações intersetoriais, mobilização do controle social. Sem dúvida, a transformação das práticas dos profissionais de saúde se constitui em grande desafio para a mudança do modelo assistencial, exigindo um acompanhamento sistemático do processo de trabalho por supervisores das secretarias municipais de saúde.

A supervisão, tradicionalmente considerada como instrumento auxiliar da gestão em serviços de saúde, foi incorporada no Brasil, a partir da década de 1970, principalmente em unidades hospitalares, devido ao seu grau de importância para o aumento da produtividade. No entanto, seu maior realce deu-se nos serviços de saúde da rede pública ${ }^{(2)}$.

A origem da palavra supervisão (do latim: super=sobre e vídeo=eu vejo) pode levar à interpretação exclusiva como inspeção. Porém, a visão moderna associa controle e educação, propõe uma relação entre parceiros, identifica práticas e instrumentos de atuação conjunta sobre o objeto de trabalho e, nesta linha, pode ser denominada "convisão", que significa gestão "co-laborativa" ou construção conjunta ${ }^{(3: 444)}$.

A supervisão é entendida como um trabalho conjunto da equipe de supervisão e da equipe de Saúde da Família, em que a primeira tem o papel de facilitar que as próprias equipes analisem suas práticas e reflitam sobre o trabalho e os resultados alcançados ${ }^{(4)}$. A supervisão é referenciada como evento e processo inerente ao trabalho coletivo realizado por profissionais especializados que prestam suporte ou apoio gerencial e técnico e que, quando bem encaminhada, determina melhor desempenho da equipe e da qualidade do serviço prestado ${ }^{(5)}$.
O processo de supervisão poderia incorporar a perspectiva pedagógica da educação permanente, que é entendida como aprendizagem no trabalho, onde o aprender e o ensinar se incorporam no cotidiano das organizações e ao trabalho ${ }^{(6)}$. Pode- se considerar como uma estratégia fundamental para a aprendizagem significativa, capaz de estimular a adesão da equipe aos processos de mudança das práticas de saúde. A educação permanente tem como objetivo a transformação das práticas profissionais e da organização do trabalho a partir da problematização do processo de trabalho.

No estudo "Ação educativa, gestão e prática: implicações para mudanças no modelo de atenção à saúde", problematiza-se a supervisão como estratégia gerencial, destacando o potencial pedagógico ${ }^{(7)}$. No caso estudado, a supervisão teve para a gestão um potencial para aumentar a governabilidade do sistema e, por outro lado, constituiu-se numa ferramenta pedagógica de apoio e fortalecimento do processo de trabalho das equipes, como canal para fortalecimento da comunicação entre trabalhadores e gestores.

A supervisão e educação permanente, neste sentido, têm o mesmo objetivo, que é a mudança do modelo de atenção através, principalmente, de mudança nas pessoas. Desta forma, as subjetividades das pessoas, constituídas de um pensar, um sentir e um agir, devem ser consideradas na atuação sobre o processo de trabalho em saúde pelo gestor e supervisor. Deve-se ter em foco, também, os microprocessos de trabalho, estimulando-se o trabalho em equipe e exercício da interdisciplinariedade ${ }^{(8)}$.

Dessa forma, podemos observar que as implicações das atividades de supervisão encontram-se questões como: assegurar um melhor ambiente de trabalho, aumentar a motivação da equipe, administrar conflitos de personalidade entre funcionários, orientar o planejamento do trabalho a ser executado pela equipe e o acompanhar a qualidade deste. Em relação à operacionalização parece ser preciso enfrentar problemas como inadequação da composição dos supervisores das equipes, dificuldade de recursos materiais, implantação de instrumentos gerenciais de organização (calendário e plano de supervisão, relatório de reunião, instrumentos de avaliação de desempenho).

Entretanto, os fatores para o desenvolvimento da supervisão como estratégia do modelo assistencial substituto vão além dos fatores políticos, dos instrumentos gerenciais de organização e avaliação serviços. O enfoque dado à estruturação da supervisão precisa, também, considerar novos enfoques da relação entre as 
intervenções individuais e coletivas, assim como entre os saberes clínicos e epidemiológicos no cotidiano das ações dos profissionais no intuito de reorientar as concepções e práticas de saúde.

Assim sendo, faz-se importante a discussão sobre as características e o padrão de funcionamento da supervisão desenvolvido pela Coordenação da Atenção Básica em relação ao processo de trabalho das equipes de saúde da família. Partimos do pressuposto de que a supervisão constitui-se um dispositivo pedagógico gerencial que, através do monitoramento e apoio pedagógico à equipe, seja capaz de contribuir para a elaboração de práticas de saúde democráticas e adequadas para o atendimento as necessidades da população, com a melhoria da qualidade da assistência prestada.

Desse modo delimitamos. inicialmente. como problema de investigação: A supervisão tem potencializado o processo de trabalho desenvolvido pelos profissionais das equipes de saúde da família?

O município em estudo passa por um processo de implementação da supervisão da atenção básica, entendendo a necessidade de sua sistematização. Pensamos que a presente pesquisa contribui para fortalecer o processo de trabalho dos profissionais das equipes de saúde da família e dos supervisores. Além disso, é possível que atribua maior valorização da supervisão como estratégia de gerenciamento, monitoramento e apoio pedagógico das práticas das equipes de saúde da família.

\section{MÉTODO}

Trata-se de uma pesquisa descritiva de abordagem qualitativa, analisando a supervisão da atenção básica em um município do Sudoeste Baiano e realizado em novembro de 2008. Foram foram participantes do estudo quatro supervisores da atenção básica (dois odontólogos e dois enfermeiros). Com estes a coleta de dados empregada foi a entrevista semiestruturada. Foram também sujeitos da pesquisa 8 profissionais (quatro enfermeiras; três odontólogos e uma médica) das equipes de saúde da família da zona urbana cuja técnica de coleta de dados foi o grupo focal. Foi critério de inclusão estar inserido em uma das equipes de saúde sob responsabilidade dos supervisores. A amostra foi intencional, dentre todas as equipes sob responsabilidade do Núcleo de Supervisão, determinada por meio de sorteio aleatório. Os profissionais dessas equipes participaram de uma sessão do grupo focal, realizado no Centro de Referência em Doenças Sexualmente Transmissíveis em uma sala com as seguintes carac- terísticas: clara, sem ruídos, afastado da interferência de terceiros e de fácil acesso para todos.

O procedimento utilizado para a coleta de dados ocorreu em três etapas: pesquisa documental em que foram utilizados os instrumentos de supervisão do município em estudo, elaborados pela equipe de supervisores da Secretaria de Saúde do município; entrevista semiestruturada com os supervisores da atenção básica; técnica do grupo focal com os profissionais das equipes da saúde da família. Foi utilizada a técnica da triangulação das informações decorrente da pesquisa documental, entrevistas e do grupo focal. Para classificação dos dados foi elaborada uma matriz de análise, dividido em objeto de estudo, categorias, roteiro para coleta de dados e fontes de análise.

A Coordenação da Atenção Básica do município, entendendo a necessidade de sistematização do processo de supervisão das equipes, construiu um organograma, redimensionando suas ações em núcleos definidos como área técnica e supervisão. A área técnica foi composta por quatro profissionais (três enfermeiros e um odontólogo) responsáveis pelas ações programáticas. O núcleo de supervisão é formado por quatro profissionais (dois odontólogos e dois enfermeiros) que acompanham as $38 \mathrm{ESF}$ e as 8 unidades tradicionais. Este tem como função garantir suporte técnico para o processo de trabalho das equipes, bem como atividades específicas como: organização de treinamento introdutório, planejamento da Pactuação Programada Integrada (PPI), atividades de capacitação, construção e implementação de protocolos assistenciais.

A supervisão tem os seguintes instrumentos institucionalizados: cronograma de supervisão, plano de supervisão, relatório de supervisão e de reunião dos supervisores, avaliação das equipes da saúde da família em relação ao processo de trabalho. Também foi construído um prontuário funcional dessas equipes, contendo informações sobre caracterização da unidade; ficha de identificação da equipe; cronograma de supervisão; planilha de cursos; cronograma de atendimento; controle de folgas, férias e cursos; atestado médico; comunicação interna; denúncias (ouvidoria); advertências; intercorrências; monitoramento e avaliação da equipe (indicadores do Sistema de Informação da Atenção Básica - SIAB); relatório de reunião. O prontuário subsidia o acompanhamento permanente da dinâmica da equipe.

O projeto foi submetido e aprovado pelo Comitê de Ética em pesquisa do Instituto de Saúde Coletiva da UFBA segundo o parecer de n. 034-08. Um "Consentimento Livre e Informado" foi solicitado 
aos informantes-chave, seguindo-se os dispostos na Resolução n.196/96 do Conselho Nacional de Saúde bem como a autorização do município para o trabalho junto às unidades de saúde.

\section{RESULTADOS}

A análise da supervisão das equipes da saúde da família levou em consideração a concepção que os entrevistados apresentam sobre supervisão, o processo de supervisão, os instrumentos utilizados, e as facilidades e dificuldades para sua realização. Na percepção e compreensão dos participantes do grupo focal a supervisão emerge como uma ferramenta de desenvolvimento profissional, trabalho conjunto, de parceria, apoio técnico, contemplando a dimensão pedagógica e não mais na visão de punição e fiscalização.

Foi mencionado, por diversas vezes, a supervisão como aceita e necessária pelos supervisionados, valorizando a atuação conjunta na busca de soluções para os problemas; destaca-se o papel da supervisão na gestão como apoiador e facilitador na resolução de problemas. Comparando sua concepção na visão dos supervisores, existe uma concordância entre estes e o entendimento dos profissionais, demonstrando uma evidência de que a supervisão é considerada importante instrumento na gestão do sistema de saúde do município. Pode-se observar que a supervisão também favorece a proximidade e o estabelecimento de vínculos como aspectos favoráveis ao desenvolvimento e avaliação do processo de trabalho.

Com relação à periodicidade da supervisão, não existe um acompanhamento contínuo. As visitas acontecem a partir de uma programação, porém, sua realização é determinada em função das prioridades ou situações emergenciais surgidas.

Percebe-se que a forma de organização da atenção básica dividida em área técnica e supervisão influenciaram a organização no processo de trabalho, além de possibilitar maior enriquecimento nas intervenções que são realizadas junto às equipes. A organização do trabalho é feita através da divisão das unidades pelos supervisores, numa média de 11 equipes por supervisor. Essa forma de organização, ao que parece, pode dificultar o acompanhamento das atividades inerentes ao processo de trabalho e também em relação à periodicidade dessa estratégia.

O processo de trabalho dos supervisores se mostrou bastante dinâmico e abrangente, apesar de não garantir periodicidade fixa. Pode-se observar que a mudança no processo de trabalho não depende apenas da organização ou reorientação dos serviços ou da prática dos profissionais sofre influências do processo de supervisão. Na investigação pode-se identificar a existência de instrumentos sistemáticos utilizados na execução das atividades dos supervisores, quer por meio da análise documental, ou das entrevistas.

Dentre as dificuldades expressas, a maioria não se relacionou ao processo de trabalho dos supervisores e sim, com problemas de infraestrutura ou político gerenciais tais como: garantia do transporte, política de Recursos Humanos, garantia do plano de cargos e salários e rotatividade de profissionais. A principal facilidade citada pelos participantes foi o fato destes planejarem a realização da supervisão, o que significa dizer que este é reconhecido como ferramenta de gestão e acompanhamento. Outra facilidade citada pelos profissionais é o fato do supervisor conhecer a dinâmica da equipe por tê-lo vivenciado na prática, ou seja, a experiência e conhecimento da realidade e do processo de trabalho favorecem o acompanhamento e a resolução dos problemas e o estabelecimento de vínculos.

\section{DISCUSSÃO}

No que se refere às dificuldades e facilidades a informação dada pelos supervisores e a não existência de um acompanhamento contínuo da supervisão foi ratificada pelos profissionais, os quais afirmam que a periodicidade da supervisão pode dificultar o acompanhamento das atividades inerentes ao processo de trabalho das equipes.

Do ponto de vista operacional, o tempo, o espaço físico e o registro devem ser considerados na atividade de supervisão. A rotina de trabalho em grupo indica frequência e ritmo diferenciados. Quando as agendas não são compatíveis ocorreram reuniões improvisadas ou esvaziadas, com informações e resultados precários. Encontros sistemáticos, com participação de todos os envolvidos no processo, é uma condição essencial ao bom desempenho do trabalho ${ }^{(5)}$.

Reforça-se aí o papel da gestão no que tange ao acompanhamento contínuo das ações, no sentido de possibilitar a adoção da proposta de supervisão como norteadora das práticas que venham a colaborar para superação das dificuldades. Ainda que, por um lado não exista uma periodicidade da supervisão, demonstrou-se, por outro lado, que o processo de trabalho é dinâmico e abrangente.

A supervisão é um processo amplo, permanente, complexo, mútuo e ativo, o qual inclui exame, conhecimento, avaliação, assessoria, ensino e informação, 
abrangendo profissionais, equipes e instituições. É atividade auxiliar fundamental para o acompanhamento regular das ações de saúde, com vistas a manter elevada qualidade dos serviços ofertados à população, bem como acompanhar seu rendimento, procurando identificar e corrigir deficiências ou distorções para a otimização da competência ${ }^{(9)}$.

Ressalta-se que os instrumentos sistemáticos utilizados para execução do processo de trabalho dos supervisores e de avaliação são fontes valiosas de informação, sem as quais a supervisão pode se tornar impertinente e ineficaz.

Existem três elementos componentes do processo de trabalho: o objeto de trabalho, os meios (instrumentos) de trabalho e a atividade (trabalho propriamente dito) ${ }^{(10)}$. O processo de trabalho é a interação recíproca destes momentos em que ocorre a transformação do objeto num produto, a partir da realização de uma atividade, utilizando-se de meios ou instrumentos de trabalho ${ }^{(11)}$.

Os resultados também apontam que o planejamento é um fator facilitador do acompanhamento e, ainda, que as mudanças no processo de trabalho precisam contar com a corresponsabilidade da gestão através da equipe de supervisão. A supervisão pressupõe uma prévia negociação, deve ser planejada, projetando momentos e processos de acompanhamento, no qual a estrutura coordenadora seja capaz de corrigir rumos junto aos parceiros, subsidiando com informações e discussões, e abrindo canais de escuta, por onde podem circular justificativas e aprendizados mútuos ${ }^{(12)}$.

\section{CONSIDERAÇÕES FINAIS}

O desafio que se impõe em romper com o modelo de atenção hegemônico, curativista e individualista na busca de implementar um modelo de atenção à saúde condizente com os princípios do Sistema Único de Saúde é um luta incansável, processual com influências múltiplas. É um processo que vai da prática do profissional, passando pelo processo de supervisão e envolve condições infraestruturais e políticos gerencias que demandam uma atitude co-responsável por parte dos diversos atores envolvidos.

Nesta perspectiva, a ESF, entre outros aspectos, representa para o município a possibilidade de envolver grande número de profissionais, supervisores e gestores no processo de reorientação do modelo de atenção à saúde. Este voltado para a melhoria do acesso, humanização do atendimento e no estabelecimento de compromisso com a comunidade, com o intuito de reorganizar o sistema local de saúde, buscando melhorar a qualidade de vida da população. A supervisão confirma-se estratégia de gestão de grande relevância para o alcance dessa finalidade processual.

Os achados deste estudo demonstram que a compreensão dos participantes sobre supervisão pode ser considerada como essencial e que ao se articularem com a equipe deverão buscar a melhoria da assistência à saúde da população e do desenvolvimento do profissional. Esse é um processo de fundamental importância como instrumento de transformação e mobilização na gestão participativa, possibilitando aos supervisionados, junto com a comunidade e com os supervisores, a estabelecer democraticamente as prioridades.

O processo de trabalho dos supervisores se apresentou dinâmico e abrangente, foram identificadas várias atividades atribuídas aos supervisores, incluindo as político-gerenciais, atividades voltadas ao processo de trabalho das equipes da saúde da família, por meio da educação permanente.

Neste estudo, a atividade de supervisão apresentou-se como uma ferramenta de gestão que demanda planejamento, envolvimento, interação, continuidade e corresponsabilidade. Esta pode ser concebida como um acompanhamento contínuo, sistemático, dinâmico, analítico e pedagógico que facilita as mudanças, intervenções e planejamento do trabalho em saúde. Nessa direção, é importante que seja construída, num esforço conjunto, entre gestores, profissionais de saúde e usuários.

\section{REFERÊNCIAS}

1. Ciampone MHT, Peduzzi M. Trabalho em equipe e trabalho em grupo no programa de saúde da família. Rev Bras Enferm. [Internet] 2000;53(n.esp.) [acesso em 15 set 2008]. Disponível: http://bases.bireme.br/cgi-bin/ wxislind.exe/iah/online/?IsisScript $=$ iah/iah.xis\&src $=g$ oogle \&base $=$ LILACS\&lang $=$ p\&nextAction $=\operatorname{lnk} \& \exp$ $\mathrm{rSearch}=302247 \&$ indexSearch $=$ ID

2. Nunes TCM. A Supervisão em Saúde: um estudo exploratório em instituições públicas no âmbito federal e estadual [dissertação]. Salvador (BA): Universidade Federal da Bahia; 1986.

3. Reis CCL, Hortale VA. Programa de Saúde da Família: supervisão ou "convisão"? Estudo de caso em município de médio porte. Cad. Saúde Pública [Internet] 2004;20(2) [acesso em 15 set 2008]. Disponível: http://www.scielo. br/scielo.php?script=sci_arttext\&pid=S0102-311X2004 000200017\&lng=pt\&nrm=iso ISSN 0102-311X. http:// dx.doi.org/10.1590/S0102-311X2004000200017 
4. Matumoto S, Fortuna CM, Mishina SM, Pereira MJB, Domingos NAM. Supervisão de equipes no Programa de Saúde da Família: reflexão acerca do desafio da produção de cuidados. Interface (Botucatu). [Internet] 2004/2005;9(16) [acesso em 15 set 2008]. Disponível: http://www.scielo.br/scielo.php?script=sci_ arttext\&pid=S1414-32832005000100002\&lng=pt\&nrm =iso ISSN 1414-3283. http://dx.doi.org/10.1590/S141432832005000100002

5. Campos CEA, Garcia J. Contribuições para a supervisão dos programas sociais com foco na família. Rev. Katál. [Internet] 2007;10(1) [acesso em 15 set 2008]. Disponível: http://www.scielo.br/pdf/rk/v10n1/ v10nla11.pdf

6. Ministério da Saúde (BR). Portaria n. 198/GM/MS, de 13 de fevereiro de 2004. Institui a Política Nacional de Educação Permanente em Saúde como estratégia do Sistema Único de Saúde para a formação e desenvolvimento de trabalhadores para o setor. Brasília: Ministério da Saúde; 2004. 14p.

7. Fontoura, MS. Ação educativa, gestão e prática: implicações para mudanças no modelo de atenção à saúde [tese]. Salvador (BA): Universidade Federal da Bahia; 2007.

8. Franco T, Merhy E. PSF: Contradições e novos desafios. Conferência Nacional de Saúde Online. Tribuna Livre. [Internet] 1999; [acesso em 15 set 2008]. Disponível: http://www.datasus.gov.br/cns/temas/tribuna/PsfTito. htm

9. Ministério da Saúde (BR). Secretaria de Modernização Administrativa e Recursos Humanos. Capacitação Pedagógica para Instrutor/Supervisor área da saúde. Brasília: Ministério da Saúde; 1989.

10. Marx K. O Capital: Crítica da Economia Política. 20a ed. São Paulo: Civilização Brasileira; 2002.

11. Paim JS. A Reorganização das práticas de saúde em Distritos Sanitários. In: Mendes EV, organizador. Distrito Sanitário: o processo social de mudança das práticas sanitárias do Sistema Único de Saúde. Rio de Janeiro: Hucitec; 1993. p. 187-220.

12. Nunes TCM, Barreto CMG. Uma proposta de Supervisão para a Diretoria de Vigilância e Controle Sanitário (DIVISA). Salvador: Secretaria da Saúde do Estado;1999. 